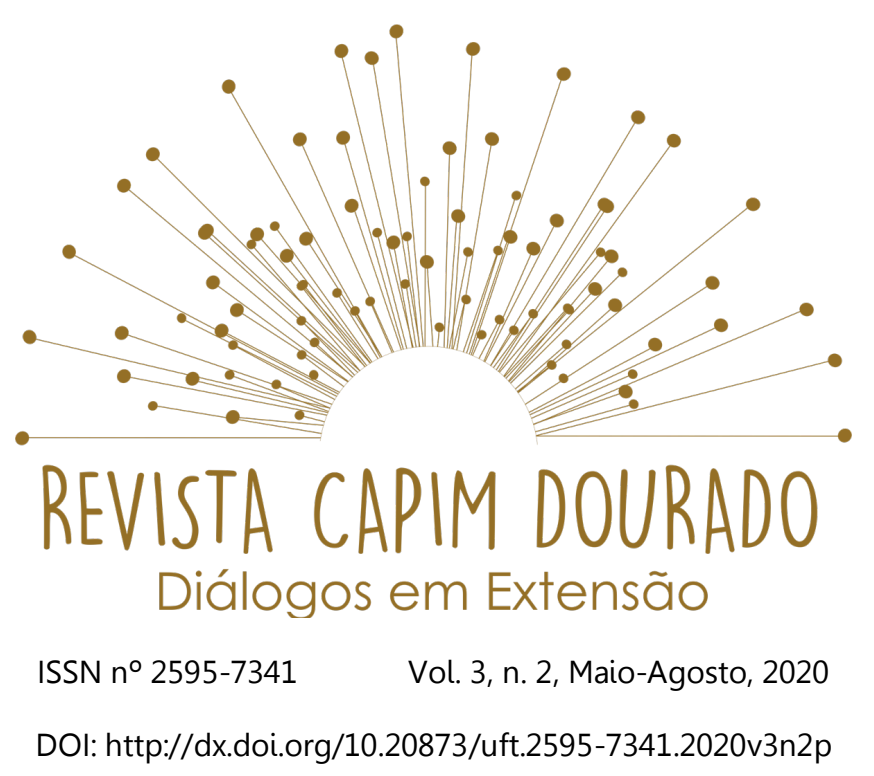

\title{
EXTENSÃO UNIVERSITÁRIA E PANDEMIA DA COVID-19: OLHARES SOBRE AS DIVERSAS CONSTRUÇÕES E FAZERES
}

A Revista Capim Dourado: Diálogos em Extensão apresenta seu segundo número de 2020. Os textos que compõem esse número focam nas práticas durante a pandemia do COVID-19 e nos ajudam a começar a caminhada e o olhar sobre a extensão em suas diversas facetas. Nesse número e no próximo, o foco serão os relatos de experiência de diversas práticas. Esses estão em espaços e momentos diferentes, demonstrando a riqueza do que a Extensão vem desenvolvendo, mesmo sob as duras situações causadas pela Covid-19 no Brasil e no mundo.

Na seção RELATO DE EXPERIÊNCIAS, temos o relato intitulado A EXTENSÃO UNIVERSITÁRIA EM TEMPOS DE PANDEMIA: a PROEX/UFT no enfrentamento da Covid-19 das autoras Josivânia Sousa Costa Ribeiro e Maria Santana Ferreira dos Santos Milhomem apresentam a extensão universitária integrada ao contexto contemporâneo e a gestão da Pró-Reitoria de Extensão, Cultura e Assuntos Comunitários-PROEX/UFT no enfrentamento da Covid-19. A extensão universitária integrada ao ensino e à pesquisa propicia a promoção de ações que articulem à interação transformadora entre universidade e sociedade corroborando com os apelos da comunidade que tanto espera da universidade, principalmente em tempos difíceis, como a pandemia.

O relato intitulado CONTRIBUIÇÕES DAS INSTITUIÇÕES DE ENSINO SUPERIOR NA EXTENSÃO UNIVERSITÁRIA EM PERÍODO DE ISOLAMENTO SOCIAL DA PANDEMIA COVID19 dos autores Francisco Gilson Rebouças Porto Junior, Meire Lúcia Andrade da Silva, Maria 


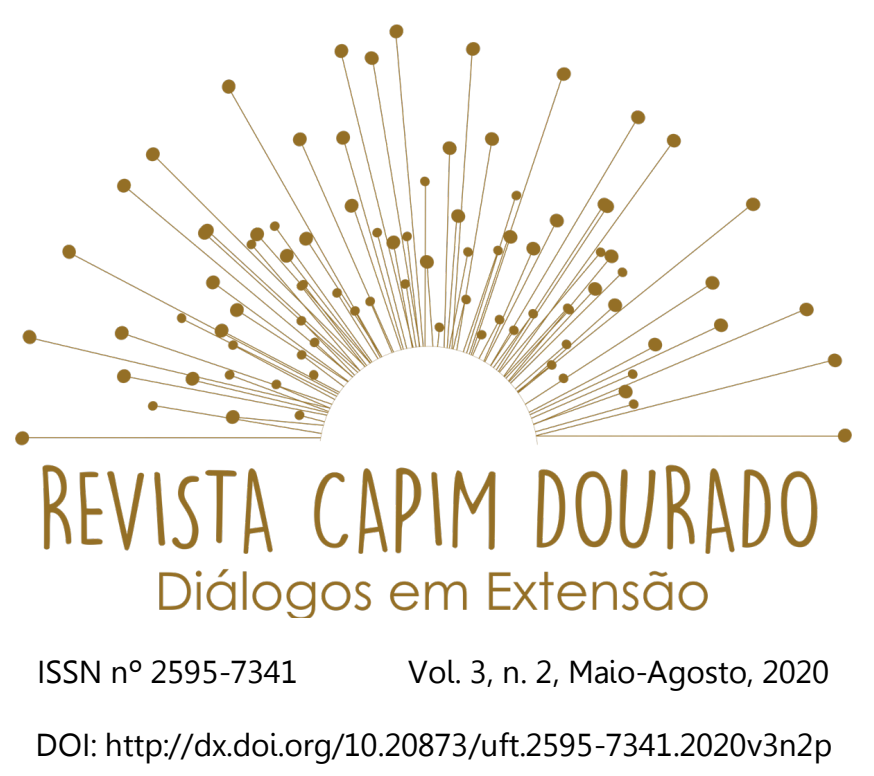

das Graças Pereira Silva, Lêda Lira Costa Barbosa abordam questionamentos na área da pandemia da COVID-19, o objetivo geral é discorrer acerca da pandemia, bem como apresentar algumas instituições que estão desenvolvendo atividades de extensão com foco na pandemia da COVID-19. Tem como questionamento que norteia esta investigação: quais as contribuições da oferta de atividades de extensão em tempos de pandemia da COVID-19? Adota-se perspectiva metodológica de pesquisa bibliográfica e documental, as fontes principais de informação são legislações pertinentes, dados e informes publicados em sites e portais. As etapas foram: escolha do tema, levantamento bibliográfico preliminar, análise textual interpretativa e problematização. Encontra-se estruturado em subtópicos que discorrem acerca da temática. Ressalta-se diante da pandemia, algumas atitudes tiveram que ser tomadas seguindo recomendações dos órgãos da saúde, das decisões políticas, administrativas, dos órgãos da gestão pública e de controle, no entanto, grande parte dessas decisões tem gerado mais incertezas do que certezas, sobretudo no meio educacional.

O relato intitulado PSICOLOGIA E EDUCAÇÃO NA PROMOÇÃO DE BEM-ESTAR PSICOSSOCIAL EM TEMPOS DE PANDEMIA: Relato de uma prática de extensão universitária dos autores Ladislau Ribeiro do Nascimento, Jayana Milhomem de Souza, Maria Santana Ferreira dos Santos Milhomem, Maria de Lourdes Leôncio Macedo relatam uma prática de extensão universitária voltada para promover saúde mental e bem-estar psicossocial no contexto da pandemia gerada pela Covid-19. Elegemos como público-alvo professores e estudantes de escolas públicas do Estado do Tocantins. Realizamos palestras e sensibilizações por meio do uso de Tecnologias da Informação e da Comunicação (TIC). Além disso, elaboramos materiais instrucionais sobre o tema em foco. $O$ trabalho resultou 


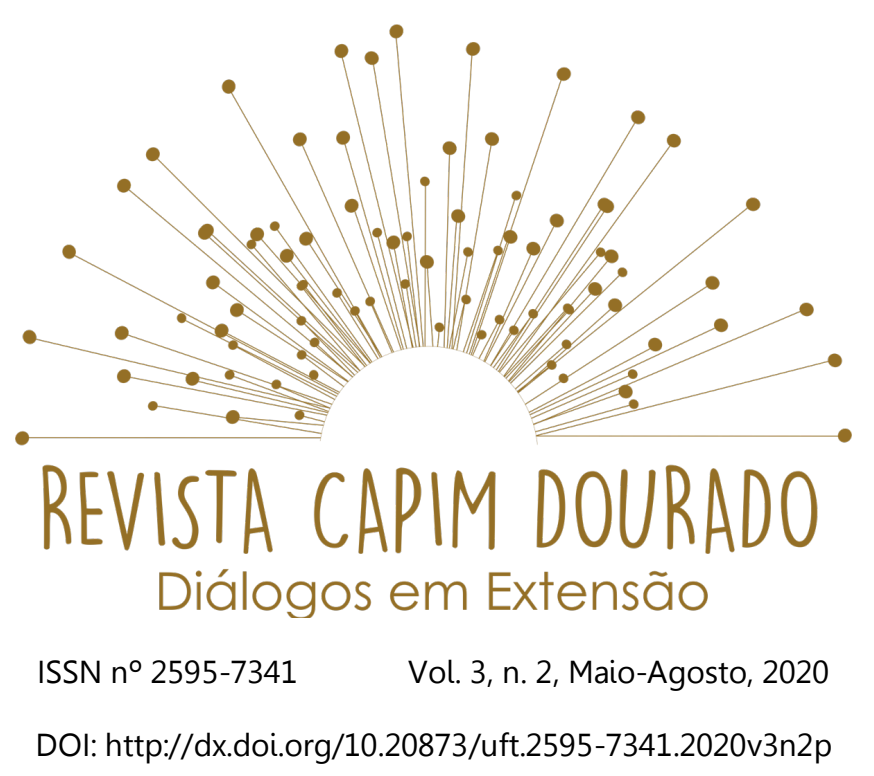

em promoção de bem-estar psicossocial e desenvolvimento de habilidades socioemocionais.

O relato intitulado CURSO COVID-19 E O PROCESSO DE TRABALHO NA ATENÇÃO PRIMÁRIA À SAÚDE: territórios, atores e diálogos dos autores Rui Massato Harayama, Juliana Gagno Lima, Edenira Nunes Costa, Julien Thalita Rocha dos Santos relatam a experiência de curso ministrado na modalidade de Ensino a Distância sobre a Covid-19 destinado a profissionais de saúde e lideranças comunitárias atendidos pela $9^{\circ}$ Região de Saúde do Estado do Pará com ênfase da Atenção Primária à Saúde no enfrentamento da pandemia.

O relato intitulado A CONTRIBUIÇÃO DA UNIVERSIDADE NA PRÁTICA EXTENSIONISTA DE ASSESSORIA A ASSISTENTES SOCIAIS QUE ATUAM NA SAÚDE NO CONTEXTO DA PANDEMIA DA COVID19 dos autores Raquel Cavalcante Soares, Delaine Melo, Ana Vieira, André França, Leila Benício, Marcelle Nogueira, Regineide Albuquerque, Renata César, Wanessa Pontes, Jefferson Silva, Julia Marques, Emily Ferreira, Julliana Araújo, Karine Lessa, Letícia Cristina de Assis, Mariana da Silva, Mariana Larissa da Silva, Thiago Henrique da Silva discutem a contribuição das ações de extensão universitária para a assessoria de profissionais de saúde, particularmente assistentes sociais, no contexto da pandemia da Covid19. O projeto de extensão em questão encontra-se em andamento, mas já há resultados importantes que evidenciam as contribuições da extensão.

O relato intitulado SER EM TEMPOS DE COVID-19: um relato de experiência de pesquisa e extensão no Departamento de Serviço Social da Universidade de Brasília dos autores Hayeska Costa Barroso, Anabelle Carrilho da Costa, Larissa Araújo Matos, Leonardo Rodrigues de Oliveira Ortegal, Taise Cristina Gomes Clementino de Negreiros, Thaís Kristosch Imperatori versam sobre o projeto de pesquisa e extensão "SER em tempos de 


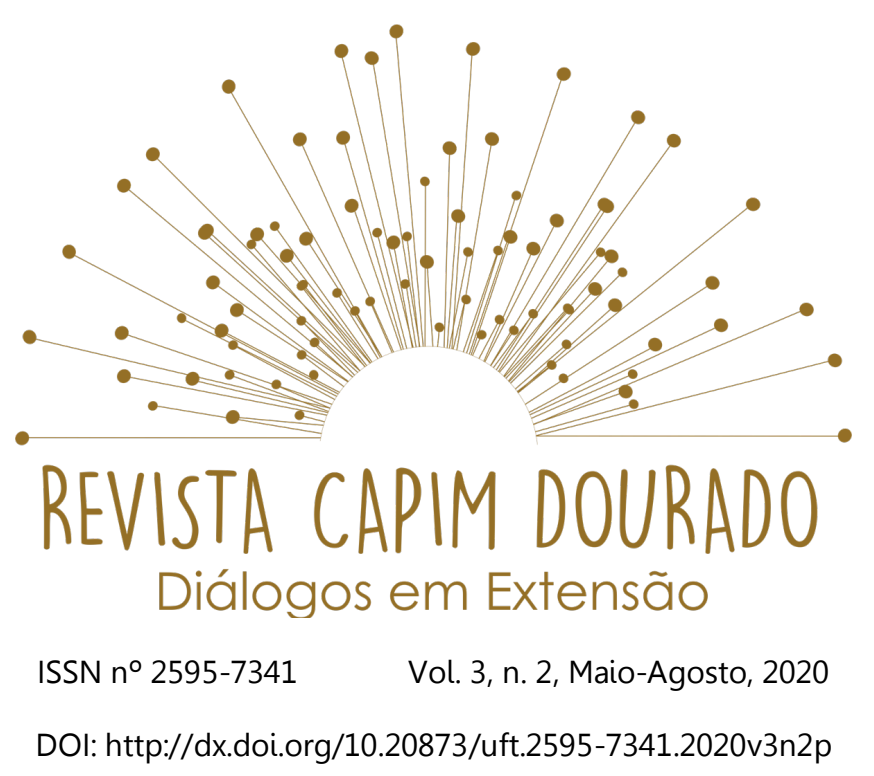

COVID 19" cujos objetivos são: socializar os dados da pesquisa sobre os desafios vivenciados pelas/os discentes do curso de Serviço Social da UnB durante a suspensão das atividades acadêmicas; construir um canal de comunicação entre o departamento, docentes e discentes e; socializar informações da universidade e produções teóricas dos/as docentes do departamento sobre o contexto de pandemia.

O relato intitulado ASPECTOS RELEVANTES DA CULTURA DO CRACK PARA A TRANSMISSÃO DA COVID-19 ENTRE CONSUMIDORES DA DROGA E POSSÍVEIS MEIOS DE PREVENÇÃO dos autores Ygor Diego Delgado Alves, Pedro Paulo Gomes Pereira, Elizângela de Freitas Silva discutem, por meio da literatura acadêmica sobre a cultura do crack, a Covid-19, notícias e entrevistas publicadas na mídia e nas redes sociais, que a disposição presente entre consumidores de crack para prevenirem riscos relacionados ao consumo da droga pôde ser agenciada, no contexto da Covid-19, por ativistas e profissionais engajados na defesa da saúde dessas pessoas com a distribuição de kits de higiene, concomitante a entrega de refeições e vestimentas. O contexto proporcionado pelos hotéis remanescentes do Programa De Braços Abertos, mesmo sem apoio da administração municipal, pode ser propício ao isolamento social contanto que esteja associado a ativistas e entidades.

O relato intitulado ATENTOS/AS E FORTES NA QUARENTENA: tecendo resistências em defesa dos direitos, das políticas sociais e das liberdades democráticas a partir da extensão universitária das autores Vanda Micheli Burginski, Rosemeire Santos sistematizam a experiência do projeto de extensão na modalidade de eventos sob o título "Atentos/as e fortes na quarentena: tecendo resistências em defesa dos direitos humanos, das políticas sociais e das liberdades democráticas, desenvolvido pelos Grupos de Estudos e Pesquisas GEPPSFAM - Grupo de Estudos e Pesquisas em Proteção Social e Famílias e o GEPSI - Grupo 


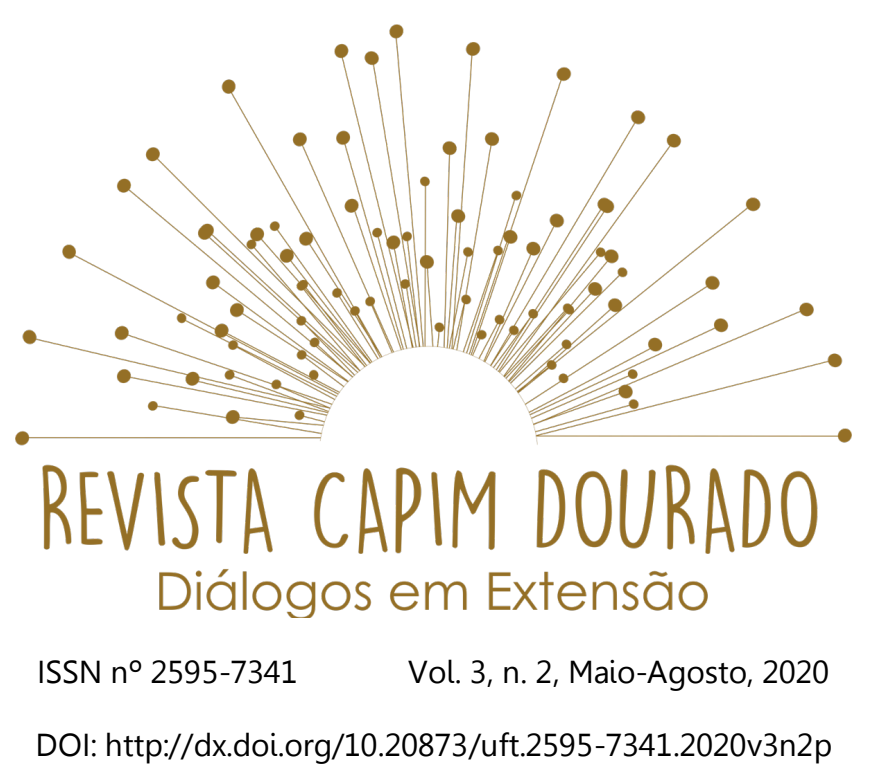

de Estudos e Pesquisas em Políticas Sociais, Intersetorialidade e Interdisciplinaridade, ambos da Universidade Federal do Tocantins (UFT) e vinculados ao curso de Serviço Social e ao Programa de Pós-graduação em Serviço Social. O projeto teve por objetivo central propiciar um espaço de debates e reflexões sobre temas relacionados ao Serviço Social, a política social e temas transversais relacionados ao impacto da pandemia da Covid-19 nas condições de vida da população, abordando as dimensões de classe, gênero, étnico-racial e geracional.

O relato intitulado ADVERSE PSYCHOLOGICAL ASPECTS OF PANDEMICS dos autores Wildson Cardoso Assunção, Weverson Cardoso de Jesus, Nathalia Stefany Pereira Carneiro apresentam o levantamento e a descrição de quais são os aspectos psicológicos adversos característicos de pandemias, tendo como referência estudos descritivos que avaliem efeitos psicológicos nas diversas populações. Este estudo é uma revisão narrativa da literatura. Essa metodologia objetiva-se proporcionar uma síntese de conhecimento através de um determinado assunto. As buscas foram realizadas nas bases de dados Biblioteca Virtual em Saúde - BVS, incluindo suas plataformas indexadas, Researchgate, Google Acadêmico, publicações de repositórios e anais de congressos. Toda a análise foi baseada na interpretação dos textos e discussão dos resultados das publicações. Em função do levantamento bibliográfico encontraram-se várias modalidades de pesquisas, variando as populações atingidas, com base nessas publicações foi possível comparar os sintomas psicológicos. Em contextos, onde há medidas mais rígidas de controle, é comum o surgimento de agravos psicológicos, leves e graves.

O relato intitulado RELATO DE EXPERIÊNCIAS DISCENTES DO NÚCLEO DE SAÚDE BUCAL INTEGRANTE DO PROJETO DE EXTENSÃO "ENFRENTAMENTO AO COVID19: produzir saúde e defender a vida das autoras L Márcia Maria Dantas Cabral de Melo, Larissa 


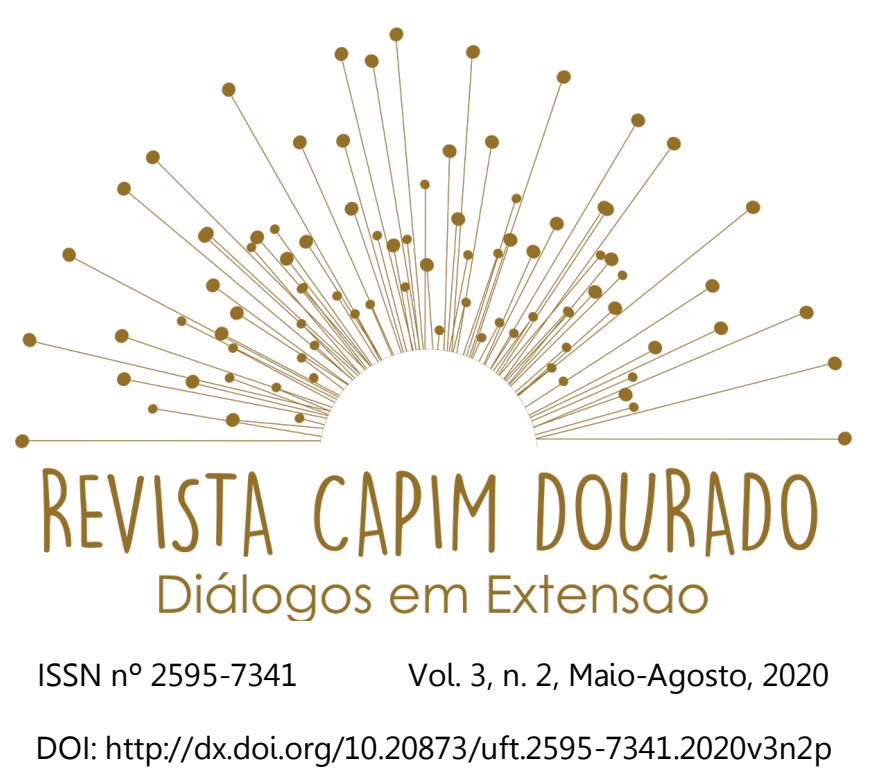

Sercundes Farias dos Anjos, Huane Soel Feitosa Rolim, Vanessa Lopes do Nascimento, Cleverton da Silva Santos, Amanda Caroline Oliveira Henriques Mendes, Sarah Marília Pereira Jordão do Nascimento trazem das ações desenvolvidas pelo núcleo de saúde bucal no Projeto de Extensão Enfrentamento ao COVID19: produzir Saúde e defender a vida da Residência em Saúde da Família da Universidade Federal de Pernambuco, sob um olhar discente. Descreve-se os objetivos, eixos de ação e as experiências iniciais do projeto vivenciadas pelo coletivo de odontologia durante a construção de materiais educativos e implementação das ações de comunicação, promoção da saúde e educação popular em saúde nas áreas de atuação da Residência na nos DS IV e V do Recife-PE. A partir das impressões iniciais, verifica-se o agir participativo discente nas primeiras ações para resistir aos impactos da pandemia nos territórios da Atenção Primária à Saúde.

O relato intitulado A OBSERVAÇÃO DO CÉU COMO ESTRATÉGIA DE APROXIMAÇÃO DE ALUNOS DO ENSINO FUNDAMENTAL E MÉDIO À TEMAS CIENTÍFICOS dos autores Ana Maria da Silva Maia, Sílvio Quintino de Aguiar Filho, Ramilla Rízzia Mira de Sousa, Bruna Lima Rocha, Alisson Soares de Sousa Santos discutem o interesse por ciências em estudantes de ensino fundamental e médio. Para isso foram efetuadas atividades relacionadas com diversas áreas da ciência, tais como química, matemática e física, utilizando a astronomia como tema transversal. As atividades foram efetuadas tanto no interior de escolas quanto em praças públicas, envolvendo não só os alunos, mas seus familiares com a importância do tema. Com isso, se estabeleceu uma relação entre a universidade e uma parcela da sociedade que pode ser diretamente beneficiada com a presença de uma instituição pública de ensino superior.

O relato intitulado DEIXA EU TE CONTAR: existe alegria no meu "arraiá" dos autores Débora Cristiana Alves Soares de Albuquerque, Maria José de Pinho, Fabíola Andrade 


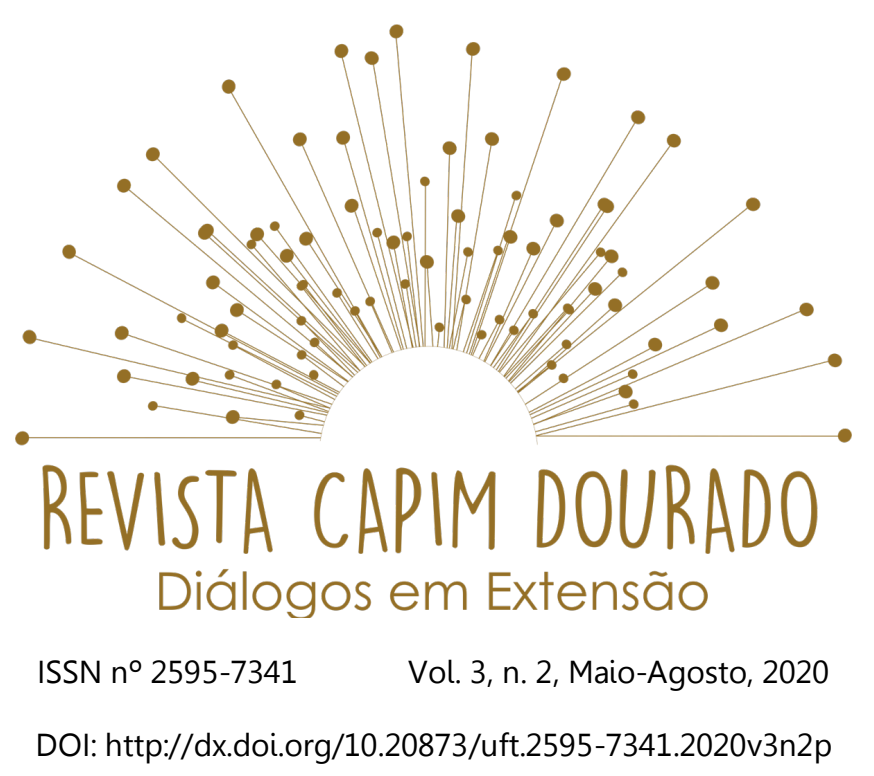

Pereira, Wellington Mota de Sousa, Marina Carla da Cruz da Cruz Queiroz relatam a vivência de uma senhora em tempos de pandemia. Essa experiência ocorreu em uma cidade do interior do Tocantins e é fruto de um Projeto de Extensão da Universidade Federal do Tocantins por meio do GATI- Grupo de Apoio a Terceira Idade. O intuito do Projeto é promover ações que elevem a autoestima e também a qualidade de vida dos idosos, demonstrando que mesmo em tempos difíceis a universidade tem conduzido ações de extensão dando visibilidade para o idoso.

O relato intitulado APOIO EMOCIONAL A CUIDADORES DE IDOSOS: vivência de voluntários do projeto escuta solidária dos autores Daniella Pires Nunes Jéssica da Silva Marinho, Ilaíse Brilhante Batista,Yana Caroline Fernandes Ferreira, Guiomar Virginia Vilela Assunção de Toledo Batello, James Stefison Sousa Santos, Bruna Mourão Moura, Adriane Ribeiro da Silva Amend, Fabiana Daronch, Loan Aragão Piva, Antônio Matheus Santos Medrado, Taísa Souza Ribeiro, Maria Sortênia Alves Guimarães, Andréia Queiroz Ribeiro relatam a vivência de voluntários em ações do Projeto Escuta Solidária voltadas aos cuidadores de idosos acamados. Os voluntários realizavam ligações telefônicas que possibilitaram o estreitamento de vínculo com cuidadores, permitindo o desenvolvimento de habilidades como o acolhimento, a escuta qualificada e o reconhecimento das necessidades do cuidador. A experiência perpassou o conhecimento científico, promovendo aos voluntários percepção de gratidão e engrandecimento na execução das ações.

E por fim, o relato intitulado AÇÃO EXTENSIONISTA NO INSTITUTO FEDERAL BAIANO CAMPUS SERRINHA: reflexões sobre um projeto experienciado durante a pandemia do COVID-19 dos autores Mariana Eloy dos Reis, Leandro dos Santos Damasceno, Erasto Viana Silva Gama apresentam a experiência do projeto "Viver Melhor" 


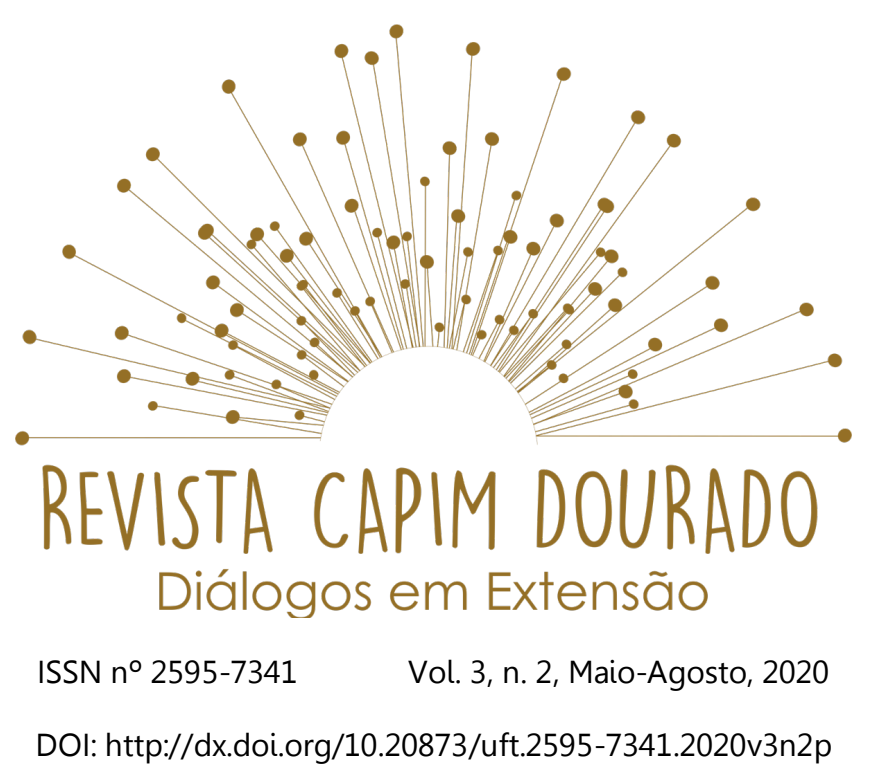

desenvolvido pelo IF Baiano Campus Serrinha, com o objetivo de dar visibilidade as ações institucionais, promover meios de inclusão social, possibilitar a envolvimento da comunidade interna e externa, durante a suspensão das atividades presenciais em decorrência da COVID-19. As ações do projeto envolveram 10 categorias de participação do público, sendo geradas até o momento 65 produções entre vídeos, cards e imagens, divulgados nas redes sociais da instituição. $O$ aprendizado tem se expandido ao longo do projeto, porém avaliação e ajustes permanentes precisam ser trabalhados para evitar fragilização do vínculo criado.

Esperamos que a leitura dos artigos e relatos amplie seu olhar sobre as possibilidades da extensão universitária.

Palmas, TO, maio de 2020.

Editor Geral / Chief Editor / Editor general

Prof. Dr. Francisco Gilson Rebouças Porto Junior, Universidade Federal do Tocantins (UFT), Brasil.

Profa. Dra. Vanda Micheli Burginski, Universidade Federal do Tocantins (UFT), Brasil.

\section{Referências}

Alves Soares de Albuquerque, D. C., Alves Soares de Albuquerque, D. C., José de Pinho, M., Andrade Pereira, F., Mota de Sousa, W., \& da Cruz Queiroz, M. C. da C. (2020). DEIXA EU TE CONTAR: existe alegria no meu "arraiá". Capim Dourado: Diálogos Em Extensão, 3(2), 190-198. Recuperado de https://sistemas.uft.edu.br/periodicos/index.php/capimdourado/article/view/9820. 


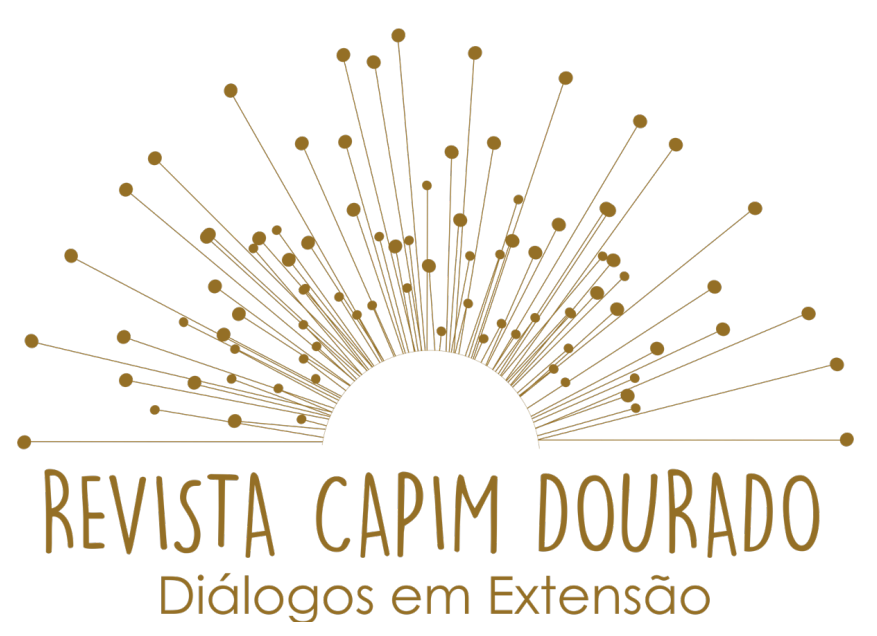

ISSN n² 2595-7341

Vol. 3, n. 2, Maio-Agosto, 2020

DOI: http://dx.doi.org/10.20873/uft.2595-7341.2020v3n2p

Barroso, H. C., Costa, A. C. da, Matos, L. A., Ortegal, L. R. de O., Negreiros, T. C. G. C. de, \& Imperatori, T. K. (2020). SER EM TEMPOS DE COVID-19: um relato de experiência de pesquisa e extensão no Departamento de Serviço Social da Universidade de Brasília. Capim Dourado: Diálogos Em Extensão, 3(2), 90-100. Recuperado de https://sistemas.uft.edu.br/periodicos/index.php/capimdourado/article/view/9809.

Barroso, H. C., Costa, A. C. da, Matos, L. A., Ortegal, L. R. de O., Negreiros, T. C. G. C. de, \& Imperatori, T. K. (2020). SER EM TEMPOS DE COVID-19: um relato de experiência de pesquisa e extensão no Departamento de Serviço Social da Universidade de Brasília. Capim Dourado: Diálogos Em Extensão, 3(2), 90-100. Recuperado de https://sistemas.uft.edu.br/periodicos/index.php/capimdourado/article/view/9809.

Burginski, V. M., \& Santos, R. (2020). ATENTOS/AS E FORTES NA QUARENTENA: tecendo resistências em defesa dos direitos, das políticas sociais e das liberdades democráticas a partir da extensão universitária. Capim Dourado: Diálogos Em Extensão, 3(2), 128-144. Recuperado de https://sistemas.uft.edu.br/periodicos/index.php/capimdourado/article/view/9975.

Burginski, V. M., \& Santos, R. (2020). ATENTOS/AS E FORTES NA QUARENTENA: tecendo resistências em defesa dos direitos, das políticas sociais e das liberdades democráticas a partir da extensão universitária. Capim Dourado: Diálogos Em Extensão, 3(2), 128-144. Recuperado de https://sistemas.uft.edu.br/periodicos/index.php/capimdourado/article/view/9975.

Cardoso Assunção, W., Cardoso de Jesus, W., \& Pereira Carneiro, N. S. (2020). ADVERSE PSYCHOLOGICAL ASPECTS OF PANDEMICS. Capim Dourado: Diálogos Em Extensão, 3(2), 145-169. Recuperado de https://sistemas.uft.edu.br/periodicos/index.php/capimdourado/article/view/9507.

Cardoso Assunção, W., Cardoso de Jesus, W., \& Pereira Carneiro, N. S. (2020). ADVERSE PSYCHOLOGICAL ASPECTS OF PANDEMICS. Capim Dourado: Diálogos Em Extensão, 3(2), 145-169. Recuperado de https://sistemas.uft.edu.br/periodicos/index.php/capimdourado/article/view/9507.

da Silva Maia, A. M., de Aguiar Filho, S. Q., Mira de Sousa, R. R., Lima Rocha, B., \& Soares de Sousa Santos, A. (2020). A OBSERVAÇÃO DO CÉU COMO ESTRATÉGIA DE APROXIMAÇÃO DE ALUNOS DO ENSINO FUNDAMENTAL E MÉDIO À TEMAS CIENTíFICOS. Capim Dourado: Diálogos Em Extensão, 3(2), 182-189. Recuperado de https://sistemas.uft.edu.br/periodicos/index.php/capimdourado/article/view/9769.

Delgado Alves, Y. D., Gomes Pereira, P. P., \& de Freitas Silva, E. (2020). ASPECTOS RELEVANTES DA CULTURA DO CRACK PARA A TRANSMISSÃO DA COVID-19 ENTRE CONSUMIDORES DA DROGA E POSSÍVEIS MEIOS DE PREVENÇÃO. Capim Dourado: Diálogos Em Extensão, 3(2), 101-127. Recuperado de https://sistemas.uft.edu.br/periodicos/index.php/capimdourado/article/view/9806.

Harayama, R. M., Gagno Lima, J., Nunes Costa, E., \& Rocha dos Santos, J. T. (2020). CURSO COVID-19 E O PROCESSO DE TRABALHO NA ATENÇÃO PRIMÁRIA À SAÚDE: territórios, atores e diálogos. Capim Dourado: Diálogos Em Extensão, 3(2), https://sistemas.uft.edu.br/periodicos/index.php/capimdourado/article/view/9829. 


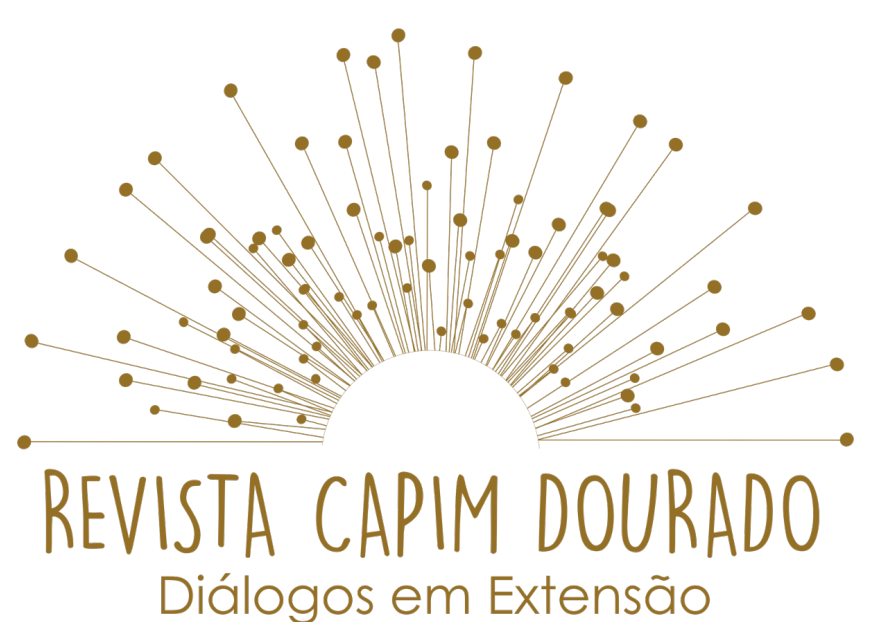

ISSN n² 2595-7341

Vol. 3, n. 2, Maio-Agosto, 2020

DOI: http://dx.doi.org/10.20873/uft.2595-7341.2020v3n2p

Melo, M. M. D. C. de, Farias dos Anjos, L. S., Feitosa Rolim, H. S., Lopes do Nascimento, V., Santos, C. da S., Mendes, A. C. O. H., \& Nascimento, S. M. P. J. do. (2020). RELATO DE EXPERIÊNCIAS DISCENTES DO NÚCLEO DE SAÚDE BUCAL INTEGRANTE DO PROJETO DE EXTENSÃO "ENFRENTAMENTO AO COVID19: PRODUZIR SAÚDE E DEFENDER A VIDA". Capim Dourado: Diálogos Em Extensão, 3(2), 170-181. Recuperado de https://sistemas.uft.edu.br/periodicos/index.php/capimdourado/article/view/9855.

Melo, M. M. D. C. de, Farias dos Anjos, L. S., Feitosa Rolim, H. S., Lopes do Nascimento, V., Santos, C. da S., Mendes, A. C. O. H., \& Nascimento, S. M. P. J. do. (2020). RELATO DE EXPERIÊNCIAS DISCENTES DO NúCLEO DE SAÚDE BUCAL INTEGRANTE DO PROJETO DE EXTENSÃO "ENFRENTAMENTO AO COVID19: PRODUZIR SAÚDE E DEFENDER A VIDA". Capim Dourado: Diálogos Em Extensão, 3(2), 170-181. Recuperado de https://sistemas.uft.edu.br/periodicos/index.php/capimdourado/article/view/9855.

Nascimento, L. R. do, Milhomem de Souza, J., Santana Ferreira dos Santos Milhomem, M., \& Leôncio Macedo, M. de L. (2020). PSICOLOGIA E EDUCAÇÃO NA PROMOÇÃO DE BEM-ESTAR PSICOSSOCIAL EM TEMPOS DE PANDEMIA: Relato de uma prática de extensão universitária. Capim Dourado: Diálogos Em Extensão, 3(2), 47-69. Recuperado de https://sistemas.uft.edu.br/periodicos/index.php/capimdourado/article/view/9859.

Nunes, D. P., da Silva Marinho, J., Brilhante Batista, I., Caroline Fernandes Ferreira, Y., Virginia Vilela Assunção de Toledo Batello, G., Stefison Sousa Santos, J., Mourão Moura, B., Ribeiro da Silva Amend, A., Daronch, F., Aragão Piva, L., Matheus Santos Medrado, A., Souza Ribeiro, T., Alves Guimarães, M. S., \& Queiroz Ribeiro, A. (2020). APOIO EMOCIONAL A CUIDADORES DE IDOSOS: vivência de voluntários do projeto escuta solidária. Capim Dourado: Diálogos Em Extensão, 3(2), 199-208. Recuperado de https://sistemas.uft.edu.br/periodicos/index.php/capimdourado/article/view/9799.

Porto Junior, F. G. R., Silva, M. L. A. da, Silva, M. das G. P., \& Barbosa, L. L. C. (2020). CONTRIBUIÇÕES DAS INSTITUIÇÕES DE ENSINO SUPERIOR NA EXTENSÃO UNIVERSITÁRIA EM PERÍODO DE ISOLAMENTO SOCIAL DA PANDEMIA COVID-19. Capim Dourado: Diálogos Em Extensão, 3(2), 30-46. Recuperado de https://sistemas.uft.edu.br/periodicos/index.php/capimdourado/article/view/9863.

Reis, M. E. dos, Damasceno, L. dos S., \& Gama, E. (2020). AÇÃO EXTENSIONISTA NO INSTITUTO FEDERAL BAIANO CAMPUS SERRINHA: reflexões sobre um projeto experienciado durante a pandemia do COVID19. Capim Dourado: Diálogos Em Extensão, 3(2), 209-219. Recuperado de https://sistemas.uft.edu.br/periodicos/index.php/capimdourado/article/view/9974.

Sousa Costa Ribeiro, J., \& Milhomem, M. S. F. dos S. (2020). A EXTENSÃO UNIVERSITÁRIA EM TEMPOS DE PANDEMIA: a PROEX/UFT no enfrentamento da Covid-19. Capim Dourado: Diálogos Em Extensão, 3(2), 2229. Recuperado de https://sistemas.uft.edu.br/periodicos/index.php/capimdourado/article/view/9798. 